\title{
Análisis de las recomendaciones sobre el tipo de apósito y la frecuencia de las curas del catéter permanente tunelizado para hemodiálisis según guías de práctica clínica
}

\author{
Ana Chiara Caro, Trinidad López Sánchez, Marta Pérez Doctor
}

Hospital Universitario Virgen del Rocío. Sevilla

\section{Introducción:}

La principal y más grave complicación del catéter permanente tunelizado (CPT) para hemodiálisis (HD) es la infección, tanto la bacteriemia (BRC) como las del orificio de salida, relacionadas directamente con la morbimortalidad, el aumento de hospitalizaciones y el gasto sanitario. Una manipulación basada en la asepsia sin medidas farmacológicas adicionales con unos protocolos estandarizados ayudan a disminuir las complicaciones y mantener las tasas de BCR dentro de la excelencia $(<1 / 1000$ días de catéter). El tipo de apósito y la frecuencia de sus cambios plantean un objetivo de nuestra necesidad de información para consensuar la mejor toma de decisiones sobre esta práctica que reduzca la variabilidad de criterios.

\section{Objetivos:}

- General:

Implantar protocolos y sistemáticas de trabajo actualizados donde la asepsia sea la principal arma de prevención de infecciones de CPT.

- Específico:

Revisar, difundir y consensuar la mejor práctica sobre: -Tipo de apósito: poliuretano transparente 0 gasa seca. -Frecuencia de las curas.

\section{Metodología:}

Base objetiva de la Enfermería basada en la Evidencia.

- Externa: Búsqueda según Guías de Práctica Clínica: web exploraevidencia en guías españolas, en Guía Salud y británicas (NICE, SING). Resto de documen- tos consultados en: Cochrane, Pub Med, IME, Cinahl y Cuiden Plus.

- Revisión de la lista de literatura encontrada con asignación del nivel de evidencia mediante clasificación GRADE (calidad, importancia relativa de los resultados y fuerza de las recomendaciones).

- Elaboración de una sesión clínica bibliográfica con lectura crítica (revisión por pares), discusión y consenso de expertos: Método de uso Apropiado (7-9 miembros: evidencia interna) y toma de decisiones.

\section{Resultados:}

Evidencia con hallazgos contradictorios y deficientes entre la descripción explícita de:

- Recomendar apósito transparente y cambio semanal para evitar curas innecesarias y potencialmente lesivas por maceración de la piel salvo sintomatología de infección o apósito sucio/despegado en que se recomienda el apósito seco. Otras revisiones actualizadas (año 2010) con Ensayos Controlados Aleatorios concluyen que "no hay suficientes datos para determinar qué tipo de apósito tiene el riesgo más bajo de infecciones pudiendo usar ambos salvo sintomatología o exudado/ apósito sucio en que se sugiere el de gasa seca. No hay conclusión sobre la frecuencia de los cambios y deben considerarse en estudios futuros.

- Convocatoria de sesión clínica con defensa evidenciada del uso de apósito transparente basada en la prevención y riesgo de lesión cutánea.

- Actualización del protocolo y la práctica clínica actual con adicción de los datos consensuados y propuestas de nuevos estudios aleatorios (proyecto de investigación prospectivo) sobre tipo y cambio de apósito. 


\section{Conclusiones:}

La revisión de la evidencia y el consenso multidisciplinar en la actualización de los protocolos sobre el manejo de los CPT contribuye en la prevención de las complicaciones infecciosas, el aumento de la vida media del acceso y la seguridad clínica del paciente con su participación activa (elección del apósito y educación en los cuidados del acceso).

\section{Referencias Bibliográficas}

1. Ruiz Seixas, Margarita. Infección asociada al catéter de Hickman en trasplante de progenitores hematopoyéticos. Relación con el periodo desde el implante al trasplante. Biblioteca Lascasas, 2009; 5(3). Disponible en http://www.index-f.com/lascasas/documentos/lc0429.php

2. Carcela Salvador, T; Gutiérrez Macías, A. Evaluación del protocolo de cura del catéter tunelizado para hemodiálisis. Documentos enfermería $n^{\circ} 44$, Dic 2011. Pag 17-21.

3. Cobo Sánchez José Luis, Sainz Alonso Rosa Ana, Vicente Jiménez Ma Yolanda, Cepa García Hortensia, Pelayo Alonso Raquel, Menezo Viadero Raquel et al. Estudio comparativo de incidencia de bacteriemias relacionadas con el catéter de hemodiálisis: bioconectores frente a conexión directa. Rev Soc Esp Enferm Nefrol [revista en la Internet]. 2011 Dic [citado 2013 Abr 25]; 14(4): 230-235. Disponible en: http://scielo.isciii.es/scielo.php?script=sci_arttext\&d=S113913752011000400004\&lng=es. http://dx.doi. org/10.4321/S1139-13752011000400004.

4. Garnica León, A; Remón Castillo, N; Santesteban de la Concepción, A; Gutiérrez García, JM; Ruiz Medrano, L; Zapatería Azcona, C; Navascués Lostado, M; González Orihuela, E. Protocolo para el cuidado del acceso vascular en pacientes con terapia renal sustitutiva (TRS). Enferm Nefrol 2012; 15 Suppl (1): 102/141.

5. Sánchez Darias, R; Foronda Perea, L; Tirados Sánchez, J; Crujeiras Pérez, R. Modificación de protocolo a favor de la disminución de tasa de infección en catéter venoso central en pacientes en hemodiálisis. Enferm Nefrol 2012; 15 Suppl (1): 17/83.

6. González Martínez, MR; Redondo Simón, MC; Caro Rodríguez, I; Ojeda Ramírez, MD; García Pé- rez, AM; Huerga García, MC; Gómez Cambronero, M; Molina Álvarez, MC; García Hita, S; Fernández Valenzuela, R; Canovas Padilla, Y. Estudio de la eficacia del sellado con taurolidina y citrato $4 \%$ del catéter para hemodiálisis en la prevención de infección y trombosis. Enferm Nefrol 2012; 15 Suppl (1): 17/83.

7. Guía de Terapia intravenosa con dispositivos no permanentes en adultos. Guías de Práctica Clínica en el SNS Ministerio de Sanidad, Servicios Sociales e Igualdad. Edición 2012.

8. Mirabet Sáez, B; Ferrándiz Martínez, MA; García Fornieles, T; Sainz Montes, M M; Martí i Monros, A; Navarro Daudén, L; Martín Martínez, R. Protocolo enfermero de cuidados de los catéteres venosos tunelizados como acceso vascular para hemodiálisis. Resultados a los 450 días. Enferm Nefrol 2012; 15 Suppl (1): 17/83.

9. Aguinaga, $A$; del Pozo, JL. Infección asociada a catéter en hemodiálisis: diagnóstico, tratamiento y prevención. NefroPlus 2011;4(2):1-10.

10. Margaret McCann, Zena EHMoore. Intervenciones para la prevención de las complicaciones infecciosas en los pacientes sometidos a hemodiálisis con un catéter venoso central (Revisión Cochrane traducida). En Biblioteca Cochrane Plus 2010 Número 1. Oxford: Update Software Ltd. Disponible en: http://updatesoftware.com (Traducida de The Cochrane Library, 2010 Issue 1 Art no. CD006894 Chichester, UK: John Wiley\&Sons. Ltd.

11. Guía SEN Acceso Vascular en hemodiálisis. 2004.

12. NDT plus (2010) Nephrology Dialysis Transplation 3: 234-246 doi: 10.1093/indtplus/sfq41. Diagnosis, prevention and treatment of haemodialysis cateter-related bloodstreaminfections (CRBSI): a position statement of European Renal Best Practice (ERBP). Raymond Vanholder, Bernard Canaud, Richard fluck, Michael Jadoul, Laura Labriola.

13. Asif A y cols. Interventional nephrology: from episodic to coordinated vascular acces care. J Nephrol 2007; 20: 399-405.

14. The introduction of every new class of antimicrobial agents is followed by emergence of resistance. By 1962, penicillin-resistant S. aureus were a major threat in hospitals and nurseries. 